\title{
Y a-t-il encore une place pour la chirurgie dans le traitement de l'infertilité masculine?
}

\author{
H. NAVRatil, N. MotTet, P. Costa
}

Service d'Urologie-Andrologie, Hôpital G. Doumergue, 5, rue Hoche 30000 Nimes

\section{RESUME}

Les progrès récents de la prise en charge biologique de l'infertilité masculine ont surtout porté sur les méthodes d'assistance médicale à la procréation. Parallèlement les techniques chirurgicales se sont améliorées, en particulier avec l'apport de la microchirurgie. Le traitement du varicocèle représente la situation où la place de la chirurgie est la moins claire. Elle ne permet pas en particulier d'obtenir une augmentation du nombre de grossesses, malgré une amélioration fréquente des paramètres du sperme. Le traitement chirurgical de la cryptorchidie ne se discute plus après échec d'un traitement hormonal. Le traitement de l'azoospermie excrétoire repose sur des anastomoses microchirurgicales nécessitant une équipe entraînée. Ses meilleurs résultats sont obtenus dans les obstructions congénitales et ne doivent pas être appréciés avant 1 an postopératoire. De même la reperméabilisation déférentielle permet d'obtenir 50\% des grossesses en cas de technique microchirurgicale parfaite. La dégradation des résultats avec le temps, impose une mise de sperme en banque de façon systématique avant la réalisation d'une vasectomie. Les obstructions des canaux éjaculateurs peuvent nécessiter le recours à des gestes endoscopiques. Enfin les microponctions épididymaires ont permis de réaliser quelques fécondations. Mais les succès de l'ICSI à partir de prélèvements chirurgicaux épididymaires ou déférentiels conduisent à élargir et à revoir considérablement les indications et les protocoles opératoires. Les indications sont à préciser, et l'analyse génétique est indispensable. Mais la qualité des conceptus obtenus reste la grande interrogation des années à venir.

Mots clés : Infertilité masculine, chirurgie infertilié, ICSI

Ces dernières années, des progrès considérables ont été effectués dans la prise en charge biologique de l'infertilité masculine principalement dans le cadre des méthodes d'assistance médicale à la procréation (AMP). Ces progrès ont surtout été marqués par la fécondation in vitro et la possibilité d'utiliser des spermes de qualité très moyenne pour obtenir un conceptus. Plus récemment encore, les méthodes de S.U.Z.I et surtout d'I.C.S.I. ont permis l'utilisation de spermes de qualité très réduite tout en améliorant le nombre des grossesses obtenues.

Parallèlement, les techniques chirurgicales s'amélioraient avec l'apport fondamental de 
la microchirurgie, mais les résultats demeurent très discutés car encore très discordants suivant les équipes $[1,2]$.

\section{TRAITEMENT DU VARICOCELE}

C'est dans ce domaine que les controverses demeurent les plus évidentes [3,4]. Ceci tient en grande partie aux incertitudes qui entourent encore cette pathologie. La définition tout d'abord n'est pas claire et l'on décrit :

- les varicocèles cliniques selon différents stades dont l'analyse demeure très subjective ainsi que l'ont montré les résultats des examens cliniques réalisés par plusieurs examinateurs [3].

- les varicocèles dits infracliniques pour lesquels le diagnostic est uniquement basé sur les données de la phlébographie ou du doppler. C'est sur cette deuxième catégorie qu'il existe les plus grandes divergences d'opinion quant au caractère pathologique du reflux isolé sans dilatation veineuse sous-jacente [5]. Nous pouvons espérer dans l'avenir que la mesure précise de la température scrotale permette une meilleure approche du rôle délétère de ces varicocèles sur la fonction épididymo-testiculaire.

L'unilatéralité ou la bilatéralité du varicocèle est un point important mais la bilatéralité est estimée entre 10 et $70 \%$ suivant les différentes publications [6].

Les techniques chirurgicales ont toutes pour but d'améliorer ou de supprimer le varicocèle, de prévenir sa récidive et de limiter les complications postopératoires, pour le coût le plus faible possible avec une durée d'hospitalisation minimale.

Plusieurs procédés sont utilisés : soit la ligature chirurgicale par voie iliaque haute ou inguinale basse avec éventuellement extériorisation complète du testicule pour lier toutes les afférences veineuses, soit une microdissection au microscope avec respect de l'artère et des lymphatiques tout particulièrement pour limiter les hydrocèles postopératoires qui sont estimées à plus de $7 \%$ des cas [7]. A l'inverse, d'autres lient en masse les veines et l'artère. La comparaison entre ces deux techniques vis-à-vis de l'artère spermatique a été effectuée sans qu'il soit démontré d'avantage majeur au respect de l'artère spermatique $[8,9]$. D'autres enfin réalisent une dissection du cordon au microscope et réalisent une anastomose entre la veine spermatique et la veine épigastrique pour respecter le drainage veineux testiculaire et traiter le reflux . Ces méthodes toutefois n'ont pas été comparées entre elles en ce qui concerne leur efficacité sur l'amélioration de la fertilité.

A côté de la chirurgie conventionnelle, se sont développées, ces dernières années, d'autres techniques :

- la ligature veineuse laparoscopique Cette technique qui comporte certains risques et qui est plus coûteuse que la chirurgie conventionnelle offre un bénéfice minime pour un risque et un coût plus élevé [3].

- l'occlusion endoveineuse qui a l'avantage de réaliser dans le même temps le diagnostic phlébographique et le traitement du varicocèle. Les comparaisons avec la chirurgie conventionnelle ne permettent pas de dégager la supériorité d'une méthode par rapport à l'autre [10].

L'analyse des résultats de la chirurgie du varicocèle sur l'amélioration de la fertilité fait également l'objet d'une controverse inépuisable. Même les études contrôlées aboutissent à des résultats divergents, démontrant soit le bénéfice de la chirurgie, soit l'absence de différence entre la simple surveillance et le traitement chirurgical [11]. L'examen de toutes les séries publiées a fait l'objet d'une synthèse dans North America d'août 1994 [4]. Les résultats de 65 études regroupant 6983 patients ont été analysés. Le pourcentage des couples ayant obtenu une grossesse est de $36,95 \%$. Ces résultats sont un argument pour les parti- 
sans de l'intervention chirurgicale comme pour ceux qui estiment que le même taux de grossesse est observé durant les 2 années qui suivent la prise en charge d'un couple infertile et chez lequel le seul facteur d'infertilité retrouvé est le varicocèle [12].

En ce qui concerne les indications des traitements enfin, un certain consensus s'est dégagé ces dernières années, pour ne pas opérer les varicocèles dont les anomalies du sperme sont trop prononcées, et surtout pour développer une chirurgie préventive chez l'adolescent lorsque la surveillance clinique met en évidence un retard de croissance du testicule du côté du varicocèle par rapport à l'autre côté.

Deux arguments majeurs sont en faveur de cette indication de la chirurgie chez l'adolescent, d'une part la récupération de la croissance testiculaire après cure du varicocèle [13], d'autre part le fait que les résultats de la chirurgie sur la fertilité semblent meilleurs lorsque le traitement est réalisé précocement [14].

En conclusion, il n'y a pas de consensus réel sur l'efficacité des traitements du varicocèle dans le but d'améliorer la fertilité masculine. S'il est habituel que l'intervention, quelle qu'elle soit, améliore les paramètres du sperme, le taux de grossesse ne paraît pas significativement augmenté.

Il importe donc dans l'avenir d'essayer de mieux comprendre par quels mécanismes le varicocèle peut entraîner une infertilité masculine et pourquoi certains hommes porteurs de varicocèles ont une fertilité normale.

La comparaison entre ces deux groupes d'hommes devrait en particulier permettre de dégager des différences en ce qui concerne la température scrotale et le profil hormonal. Une meilleure compréhension de la physiopathologie des varicocèles permettrait de mieux sélectionner les patients susceptibles d'être réellement améliorés.
Il serait également important que les chirurgiens comparent l'efficacité des techniques chirurgicales entre elles de façon à définir le geste paraissant le plus efficace. Enfin, il faudrait améliorer la qualité des travaux de recherche clinique en essayant de réaliser des études randomisées avec des groupes témoins qui ne soient pas discutables.

\section{TRAITEMENT DES CRYPTORCHIDIES}

La relation entre la cryptorchidie et l'infertilité masculine n'est plus à démontrer. Deux facteurs contribuent à aggraver le pronostic : la bilatéralité de l'affection et le niveau de rétention des testicules. en particulier lorsque les testicules ne sont pas palpables. Le traitement hormonal doit impérativement être mis en ouvre avant toute chirurgie, mais en cas d'échec, la chirurgie n'est pas discutable. Le moment de l'intervention demeure toutefois un sujet de controverse. L'analyse des quelques données provenant d'études rétrospectives permet de suggérer une corrélation entre l'âge de l'intervention et la fertilité ultérieure et de proposer l'abaissement testiculaire avant l'âge de deux ans $[15,16]$.

L'acte chirurgical comporte un risque iatrogène et tout doit être mis en œuvre pour que les avantages de la chirurgie l'emportent sur les inconvénients. Un travail coopératif d'un groupe de chirurgiens pédiatriques a mis en évidence sans ambiguité l'intérêt du traitement laparoscopique qui permet une exploration précise et atraumatique des testicules cliniquement impalpables et qui prépare le traitement chirurgical dans les meilleures conditions lorsque celui-ci s'avère nécessaire [17].

Un autre point de controverse est la chirurgie des testicules oscillants pour lesquels il n'existe actuellement aucun argument dans la littérature permettant de proposer une orchidopexie [18]. 
Enfin, la chirurgie de la cryptorchidie chez l'adulte a permis, dans des cas isolés, d'obtenir une amélioration spectaculaire de la fertilité.

\section{CHIRURGIE DE L'AZOOSPERMIE EXCRETOIRE}

De nouvelles techniques ont contribué à améliorer les résultats de la chirurgie de l'obstruction épididymaire et les anastomoses épididymo-déférentielles latéro-latérales appartiennent au passé. La microchirurgie dans les mains d'opérateurs entraînés a permis de réaliser des anastomoses entre le tubule épididymaire et la lumière déférentielle en utilisant essentiellement 2 procédés techniques : l'anastomose terminoterminale et l'anastomose termino-latérale $[19,20]$. Globalement, ces deux techniques donnent les mêmes résultats.

Les améliorations ne dépendent pas seulement de la technique utilisée, mais aussi d'une meilleure sélection des indications; c'est ainsi que les meilleurs résultats seront obtenus lorsqu'après incision du tubule épididymaire, il existe un écoulement abondant contenant des spermatozoïdes mobiles. Si par contre, on réalise l'anastomose sur un tubule épididymaire où l'écoulement est peu abondant avec peu ou pas de spermatozoïdes immobiles, on diminue les chances d'obtenir un bon résultat. Dans les meilleures mains et avec des indications bien sélectionnées on obtient actuellement la présence de spermatozoïdes dans l'éjaculat dans plus de $60 \%$ des cas et un pourcentage de grossesses de plus de $20 \%$ [21].

Ainsi, 1 homme sur 5 présentant une obstruction épididymaire, a la possibilité d'avoir un enfant par procréation naturelle, ce qui justifie pleinement de poursuivre dans cette voie à la condition que cette chirurgie soit réalisée par un chirurgien ayant un entraînement permanent à la microchirurgie.
L'analyse des séries publiées fait apparaitre 2 notions importantes :

- les résultats sont meilleurs dans les obstructions d'origine congénitale ;

- l'évaluation du résultat d'une anastomose épididymo-déférentielle nécessite un recul d'un an car la survenue de spermatozoïdes dans le sperme peut être retardée [22]. C'est seulement après ce délai que l'on peut envisager une éventuelle réintervention.

Une question intéressante demeure d'actualité : les anastomoses épididymo-déférentielles doivent-elles être réservées aux seuls cas d'azoospermies excrétoires ? Un certain nombre de patients, en effet, ont une période d'oligo-asthénotératospermie excrétoire avant de parvenir au stade d'azoospermie, ce fait étant retrouvé dans un bon nombre de stérilités secondaires. La présence de testicules cliniquement normaux, éventuellement une biopsie testiculaire normale, une diminution du taux des marqueurs épididymaires sont autant d'éléments qui peuvent conduire à envisager une chirurgie de l'oligo-asthénospermie d'autant que l'on réalisera au cours de cette intervention un prélèvement de spermatozoïdes en vue d'une assistance médicale à procréation ou d'une congélation [23]. Toutefois, les cas publiés sont encore en nombre trop faible pour pouvoir prendre clairement position sur cette indication particulière de la chirurgie.

\section{CHIRURGIE DE REPERMEABILISA- TION DES CANAUX DEFERENTS APRES VASECTOMIE}

Les résultats de cette chirurgie sont particulièrement bien étudiés aux Etats-Unis où la vasectomie intéresse environ 500000 hommes par an. Là encore, les techniques de microchirurgie ont singulièrement amélioré la qualité du résultat. Entre les mains de chirurgiens entraînés, la présence de 
spermatozoïdes dans le sperme est obtenue dans $90 \%$ des cas et des grossesses dans près de la moitié des cas. Des travaux récents et en particulier ceux de l'O.M.S. et de Goldstein [24] ont mis en évidence la détérioration de la qualité du résultat avec le temps. C'est pourquoi il est prudent, dans un pays comme la France, où il est relativement facile de mettre son sperme en banque, de demander aux patients une congélation du sperme avant la vasectomie.

\section{CHIRURGIE DE L'OBSTRUCTION DES CANAUX EJACULATEURS}

Les indications de cette chirurgie sont rares mais néanmoins intéressantes. Chaque fois qu'un homme présente une oligospermie prononcée ou une azoospermie associée à une hypospermie avec abaissement du taux de fructose, une échographie de la prostate et des vésicules séminales par voie endorectale éventuellement associée à une ponction d'un kyste de l'utricule doit être réalisée.

L'opacification des voies séminales en vue de contrôler leur intégrité et leur perméabilité peut également s'avérer utile (ponction du canal déférent ou des vésicules séminales). La chirurgie endoscopique de ces obstructions est désormais bien codifiée et permet d'obtenir une grossesse dans près de la moitié des cas [25].

\section{LES PRELEVEMENT EPIDIDYMAIRES EN VUE D'UNE FECONDATION IN VITRO}

Ces dernières années, de nombreuses équipes ont réalisé des prélèvements épididymaires en vue de réaliser des fécondations in vitro "d'indication masculine". Ce geste peut être effectué isolément ou suivi d'une éventuelle anastomose épididymodéférentielle lorsqu'elle est possible. Mais le plus souvent, il est réalisé chez des patients pour lesquels on ne peut espérer une procréation naturelle (malade paraplégique par exemple) ou chez lesquels une obstruction ou une absence des canaux déférents ne permettent pas d'envisager une anastomose épididymo-déférentielle.

Malheureusement, ces prélèvements épididymaires pour fécondation in vitro ont donné lieu à la survenue de très peu de grossesses de même que l'utilisation de sperme congelé prélevé lors d'une intervention pour exploration d'une azoospermie. Ces protocoles sont d'organisation très lourde et la prise en charge d'un prix élevé. La possibilité actuelle de réaliser une ICSI dans de telles situations avec des résultats très encourageants devrait conduire à l'abandon des FIV avec prélèvement épididymaire [26].

\section{LES PRELEVEMENTS EPIDIDYMAIRES OU TESTICULAIRES EN VUE D'I.C.S.I.}

Le succès des procréations par injection directe d'un spermatozoïde dans l'ovocyte permet de recruter des hommes chez lesquels il n'y avait plus aucun espoir en raison du nombre trop faible de spermatozoïdes recueillis. Le seul fait que l'on puisse réaliser une fécondation avec un seul spermatozoïde vivant, voire une spermatide ouvre un champ d'investigation considérable et une nouvelle voie dans le traitement de l'infertilité masculine. Ces progrès récents nous conduisent donc à revoir complètement le champs des indications et celui des protocoles opératoires [27-29].

Ainsi, lorsque la qualité du sperme ne permet pas d'espérer une procréation naturelle, il devient maintenant possible d'envisager pratiquement dans tous les cas une exploration chirurgicale en vue d'une I.C.S.I.. Des situations que l'on considérait autrefois comme sans recours, par exemple celles des hommes avec petits testicules et FSH élevée, peuvent être actuellement explorées. Dans plus de la moitié des cas où 
il existe une FSH élevée, on retrouve la présence de spermatozoïdes dans les testicules [30]. Même dans les syndromes de "cellules de Sertoli only" Silber a montré que la recherche de petits foyers de spermatogenèse pouvait être effectuée à condition d'explorer les deux testicules de façon très complète [31].

Face à cet immense espoir, il importe maintenant que les prochaines années soient celles de l'évaluation de la qualité du conceptus obtenu dans ces situations extrêmes de façon à dégager sans ambiguïté les éventuelles contre-indications. En attendant la prudence s'impose, particulièrement dans les situations où l'origine de l'infertilité masculine n'est pas claire. Une enquête génétique et un caryotype demeurent des examens indispensables avant la décision chirurgicale. Quant à la situation particulière des azoospermies par agénésie déférentielle, elles pose maintenant de façon concrète le problème de l'exploration génétique en vue de déterminer le risque de transmettre une mucoviscidose.

Une question se pose enfin: l'exploration chirurgicale en vue d'une I.C.S.I. peut-elle être abandonnée au profit d'une ponction épididymaire ou testiculaire? Quelques études contrôlées ont montré que la ponction épididymaire et surtout testiculaire permettait d'obtenir des spermatozoïdes dans les mêmes conditions que celles d'un recueil au cours d'une intervention ouverte $[32,33]$. Néanmoins, 2 raisons s'opposent pour l'instant au principe de la généralisation de ces méthodes de ponction :

- d'une part, le fait que l'exploration chirurgicale réalisée par un chirurgien entraîné permet à coup sûr de ne pas laisser passer l'indication d'une anastomose épididymo-déférentielle, ce qui reste une priorité avant d'envisager une assistance médicale à la procréation

- d'autre part, le fait que le recueil de spermatozoïdes par voie chirurgicale est plus abondant que par simple ponction ce qui permet une congélation en prévision de tentatives ultérieures.

\section{CONCLUSION}

Les résultats discutables du traitement chirurgical de certaines infertilités masculines, particulièrement ceux des anastomoses épididymo-déférentielles ont pu faire douter de son bien fondé.

Les progrès de la microchirurgie, une meilleure sélection des indications, une vraie spécialisation des chirurgiens de l'infertilité et une meilleure évaluation des résultats ont contribué à améliorer la qualité des résultats. Cette chirurgie conserve donc une réelle utilité dans la prise en charge de l'homme infertile.

Plus récemment, les résultats obtenus grâce à l'ICSI, redonnent au chirurgien andrologue une place privilégiée au sein des équipes de PMA. Il lui appartient en effet de fixer les indications de la chirurgie et de prélever des spermatozoïdes chaque fois que la situation clinique laisse penser qu'il n'y a aucun espoir de procréation naturelle [34].

\section{REFERENCES}

1. V. IZARD, B. VOG'T AND A. JARDIN : Epididymal microsurgery techniques and results. Front. in Endocrin. Vol 11, 245-258, 1995.

2. N.Mottet-Auselo, P.Costa, P.Fourquet-Canonne, H.NAVRATIL : Chirurgie de la voie séminale : évaluation critique. Progrès en Androl. 5, Editions Doin, 151-168.

3. T.B. HARGREAVE : Debate on the pros and cons of varicocele treatment in favour of varicocele treatment. Hum. Reprod. Vol 10, suppl 1, 151-157.

4. M.H. SCHLESINGER, I.F. WILETS, AND H.M. NAGLER : Treatment outcome after varicocelectomy. A critical analysis. Urol.Clin.of North Amer. Vol 21, $n^{\circ} 3$, 517-529, August 1994.

5. J.W.P. MARSMAN AND R.SChats : The subclinical varicocele debate. Hum. Reprod. Vol 9, $\mathrm{n}^{\circ} 1,1-8$, 1994. 
6. M. GOLDSTEIN : Surgical therapy of male infertility. Journ.Urol. Vol 149, 1374-1376, May 1993.

7. M. Goldstein, B.R. GilberT, A.P. DiCker, J. Dwosh AND C. GNECCO : Microsurgical inguinal varicocelectomy with delivery of the testis : an artery and lymphatic sparing technique. Journ. Urol. Vol 148, 1808-1811, december 1992.

8. T. Matsuda, Y. Hori and O. Yoshida : Should the testicular artery be preserved at varicocelectomy? Journ. Urol. Vol 149, 1357-1360, May 1993.

9. M. Yamamoto, Y. Tsujt, M. Ohmura, H. Hibi and K. MTYAKE : Comparison of artery-ligating and arterypreserving varicocelectomy : effect on post-operative spermatogenesis. Andrologia 27, 37-40 (1995).

10. E. NieschlaG, H.M. BEHRE, A. SChLINGHEIDER, D. NASHAN, J. PoHL AND A.R. Fischedick : Surgical ligation vs. angiographic embolization of the vena spermatica : a prospective randomized study for the treatment of varicocele-related infertility. Andrologia 25, 233-237, 1993.

11. E. Nieschlag, L.Hertle, A.Fischedick and H.M.BeHRE : Treatment of varicocele : counselling as effective as occlusion of the vena spermatica. Hum. Reprod. Vol 10, n² 2, 347-353, 1995.

12. BaKer HWG, Burger HG, DeKrester DM, et al : Testicular vein ligation and fertility in men with varicoceles : BMJ 291, 1678, 1995.

13. O. ATASSI, E.J. KASS AND B.W. STEINERT : Testicular Growth after successful varicocele correction in adolescents : Comparison of artery sparing - Techniques with Palomo procedure. J. Urol. Vol 153, 482-483, February 1995.

14. F. Comhatre, A. Zalata and F. Schoonjans : Varicocele : indications for treatment. Intern. J. of Androl., 18, Suppl.2 : 67-71 (1995).

15. C. Sultan, S. Lumbroso: Cryptorchidie de l'enfant : syndrome ou symptôme? Andrologie Vol $5, \mathrm{n}^{\circ} 3,309-316$, Septembre 1995.

16. R.Mieusset, L.Bujan, G.Mansat, A.Mansat and F.PONTONNIER : Clinical and biological characteristics of infertile men with a history of cryptorchidism. Hum.Reprod., Vol 10, n³, 613-619, 1995.

17. PH. VAysse : Laparoscopy and Impalpable Testis A prospective Multicentric Study (232 Cases) : Eur.J. Pediatr. Surg. 4, 329-332 (1994).

18. M. Nistal, M.D., R. Paniagua, Ph.D. : Infertility in adult males with retractile testes. Fertil. Steril. Vol 41, n³, Marc 1984.

19. M. Nistal, M.D., R. Paniagua, Ph.D. : Microscopic vasoepididymostomy : specific micro-anastomosis to the epididymal tubule. Fertil. Steril., $30: 565$, 1978.
20. J.L. MARMAR : Management of the epididymal tubule during an end-to-side vasoepididymostomy. J.Urol., Vol 154, 93-96, July 1995.

21. P.N. Schlegel and M. Goldstein : Microsurgical vasoepididymostomy : refinements and results. J. Urol., Vol 150, 1165-1168, October 1993.

22. G.J. Matthews, P.N. Schlegel and M. GoLdstein : Patency following microsurgical vasoepididymostomy and vasovasostomy : temporal considerations. Journ. Urol. Vol 154, 20702073, December 1995.

23. R. Hauser, P.D. Temple-SMith, G.J. Southwick, J. McFarlane AND D.M. DE KRETSER : Pregnancies after microsurgical correction of partial epididymal and vasal obstruction. Hum. Reprod. Vol 10, $n^{\circ} 5$, 11.52-1155, 1995.

24. A.M. Belker, A.J. Thomas, E.F. FuchS, J.W. KonNAK AND I.D. SHARLIP : Results of 1,469 microsurgical vasectomy reversals by the vasovasostomy study group. Journ. Urol. Vol 145, 505-511, march 1991.

25. R.B. MEACHAM, D.K.HeLLeRSTEIN, L.I. LIPShULTZ : Evaluation and treatment of ejaculatory duct obstruction in the infertile male. Fertil. and Steril., Vol 59, $\mathrm{n}^{\circ} 2,393-397$, february 1993.

26. S.J. Silber, Z.P. NaGy, J. Liu, H. Godoy, P. DeVRoey and A.C. VAN SteIrTEGHem : Conventional in-vitro fertilization versus intracytoplasmic sperm injection for patients requiring microsurgical sperm aspiration. Hum. Reprod. Vol 9, n ${ }^{\circ}$, 1705-1709, 1994.

27. Palermo G., Joris H., Devroey P. and Van SteirTEGHEM A. (1992) : Pregnancies after intracytoplasmic sperm injection of single spermatozoon into an oocyte. Lancet, 340, 17-18.

28. Van Steirteghem A.,LiU J., Joris H., Nagy Z.,Janssenswillen C.,Tournaye H.,Derde M-P., Van Assche E. And Devroey P. (1993a) : Higher success rate of intracytoplasmic sperm injection than by subzonal insemination. Report of the second series of 300 consecutive treatment cycles. Hum. Reprod., 8, 1055-1060.

29. Van Steirteghem A.,Nagy Z., Joris H.,LiU J.,Staessen C.,Smitz J., Wisanto A. and Devroey P. (1993b) : High fertilization rates after intracytoplasmic sperm injection. Hum. Reprod. 8, 10611066.

30. R.C. MARIN-DU-PAN AND P.BISChoF : Increased follicle stimulating hormone in infertile men. Hum.Reprod. Vol 10, nº, 1940-1950, 1995.

31. S.J.Silber, A. Van Steirteghem and P.Devroey : Sertoli cell only revisited. Hum. Reprod. Vol 10, $\mathrm{n}^{\circ} 5,1033-1036,1995$. 
32. S.J. Silber, A.C. Van Steirteghem, J. Liu, Z. Nagy, H. Tournaye and P. Devroey : High fertilization and pregnancy rate after intracytoplasmic sperm injection with spermatozoa obtained from testicle biopsy. Hum. Reprod. Vol 10, nº 1, 148-152, 1995.

33. O. Hovatta, J. Mollanen, K. von Smitten and I. REIMA : Testicular needle biopsy, open biopsy, epididymal aspiration and intracytoplasmic sperm injection in obstructive azoospermia. Hum. Reprod. Vol 10, nº 10, 2595-2599.

34. F. Comhaire, A. Zalata, A. Mahmoud, B. DepoorTER, L. HuYsse, A. Christophe AND C. DepuydT : Diagnostic and therapeutic approach to moderate and severe male subfertility in 1995. Hum. Reprod. Vol 10 Suppl.1, 144-150, 1995.

\section{ABSTRACT}

\section{H. Navratil, N. Mottet, P. Costa}

Considerable progress has been recently made in the biological treatement of male infertility. In parallel surgical techniques improved with the main impact of microsurgery. Varicoceles represent the pathology where surgery has the more questionable place. In fact it usualy increases the quality of the sperm, but has no impact on the pregnancy rate. Surgery is unquestionable for cryptorchidism after a failure of the hormonal treatment. Surgical treatment for excretory azoospermia is based on microsurgery performed by a well trained team. The results are better when the obstruction has a congenital origin, and a year is requiered for the clinical evaluation. Microsurgery allows to obtain a 50\% prenancy rate after vasovasostomy performed after a vasectomy. But a sperm banking must be systematically done before vasectomy, due to the deterioration of the quality of the restults with time. The ejaculatory duct obstruction may lead to endoscopic procedures. Finally epididymal or vas sperm collection has allow to obtain some pregnancies. But the success of ICSI from surgical epididymal or deferential fluid calls for a completely new approach to the range of indications and surgical protocols. The indications must be clearly defined, the need for a genetic screening is mandatory. And the quality of the obtained pregnancies remains the main question for the next years.

Key words : male infertility, surgery of male infertility, ICSI. 